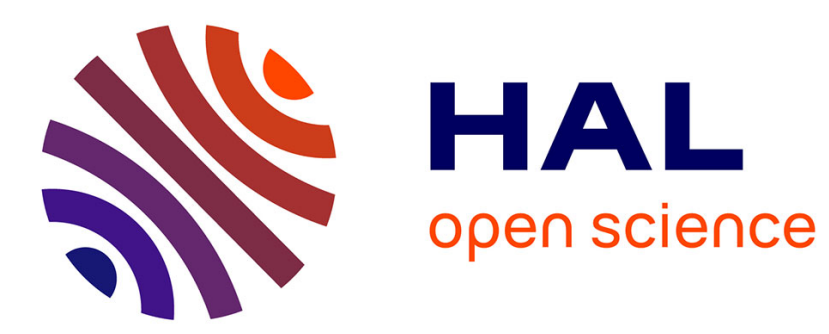

\title{
Impact of GNSS receiver tuning on the estimation of scintillation index
}

S. Rougerie, M. Ait-Ighil, V. Fabbro

\section{To cite this version:}

S. Rougerie, M. Ait-Ighil, V. Fabbro. Impact of GNSS receiver tuning on the estimation of scintillation index. ION GNSS 2016, Sep 2016, PORTLAND, United States. hal-01404423

\section{HAL Id: hal-01404423 \\ https://hal.science/hal-01404423}

Submitted on 28 Nov 2016

HAL is a multi-disciplinary open access archive for the deposit and dissemination of scientific research documents, whether they are published or not. The documents may come from teaching and research institutions in France or abroad, or from public or private research centers.
L'archive ouverte pluridisciplinaire HAL, est destinée au dépôt et à la diffusion de documents scientifiques de niveau recherche, publiés ou non, émanant des établissements d'enseignement et de recherche français ou étrangers, des laboratoires publics ou privés. 


\title{
Impact of GNSS receiver tuning on the estimation of scintillation index
}

\author{
S. Rougerie ${ }^{1}$, M. Ait-Ighil ${ }^{2}$, Vincent Fabbro ${ }^{2}$ \\ ${ }^{1}$ CNES: RF/ITP, Toulouse, France \\ ${ }^{2}$ ONERA - The French Aerospace Lab: DEMR, Toulouse, France
}

\begin{abstract}
When crossing the ionosphere layer, GNSS signals may be impacted by scintillation, a dynamic effect which causes very fast variations of the amplitude and the phase. The GNSS receivers may lose lock, which lead to unavailability of the satellite link as well as the reduced reliability of the available information. In the present state of the art, the understanding of scintillation phenomena is still insufficient, and the available models are still not adequate for GNSS system design. Thus, many studies are carried out in order to model scintillation [1] [2] [3] and evaluate the effect on the GNSS receivers and augmentation system [4] [5]. These studies monitor the scintillation thanks to specific scintillation index. However, the estimation of these scintillation indices may be affected by the receiver itself and by the estimator. The goal of this paper is to understand how receiver or estimator tuning may impact the estimation of scintillation index. To conclude, this paper gives recommendation on how to tune GNSS receiver or estimator in order to minimize their impact.
\end{abstract}

\section{INTRODUCTION}

When crossing the ionosphere layer, GNSS signals are impacted by two kinds of effects: mean effects and dynamic effects. Mean effects encompass the group delay and the signal phase advance occurring when the signal is going through the ionosphere. This delay depends on the TEC (Total Electron Content) of the ionosphere layer. It leads to a positioning error of several meters to tens of meters. Most of this delay can be corrected thanks to a dual frequency measurement, or using correction models (like Klobuchar, NeQuick or TEC grids in the case of augmentation systems) for single frequency measurements. Most of the dynamic effects are coming from the scintillation phenomena. These events generally occur over the equatorial and polar regions, but they may also occur at middle latitudes when the solar activity increases as for solar maximum. These events impact the signal and cause very fast variations of the amplitude and the phase. Therefore, the signal fades and GNSS receivers may lose lock. As a consequence, the main impact of the ionosphere dynamic effects on GNSS is the unavailability of the satellite link as well as the reduced reliability of the available information. In the present state of the art, the understanding of scintillation phenomena is still insufficient, and the available models are still not adequate for GNSS system design.

Thus, many studies are carried out in order to model scintillation [1] [2] [3] and evaluate the effect on the GNSS receivers and augmentation system [4] [5]. These studies monitor the scintillation thanks to specific indices such as ROTI (Rate Of TEC Index), S4, SigmaPhi or the slope of the scintillation spectrum. However, the estimation of these scintillation indices may be affected by the receiver itself. Indeed, ROT (Rate Of TEC) spectrum and phase spectrum are estimated thanks to the PLL and DLL outputs, which are directly affected by the loop bandwidth and loop order. Thus, the scintillation indices values, which are the results of the spectrum integration (for example ROTI is obtained by integrating ROT spectrum), may depend of the receiver tuning, and also of the integration parameters (i.e. integration time and sampling frequency).

The goal of this paper is to understand how receiver / estimator tuning may impact the estimation of scintillation indices. The approach is the following: based on a theoretical model of scintillation [2] [3], phase and amplitude time series are generated. These times series feed a GNSS receiver simulator, which at the end generate the traditional GNSS observable: pseudo-range and phase estimations. Based on these observables, conventional scintillation estimators are applied. The simulated and the estimated scintillation spectrum (and thus the scintillation indices by integration) are finally compared in order to evaluate the GNSS receiver influence. The paper is therefore organized as follow: will be presented in section II, a short review of the scintillation model, the GNSS simulator and the scintillation algorithms estimator. In the section III, the entire tests will be presented in order to evaluate the impact of the receiver on the phase and amplitude scintillation spectrum and indices. Finally, the section IV summarise all the receiver and estimator effects, give recommendation to minimise it, and present our conclusions and future work. 


\section{Scintillation simulator}

For the purpose of this study, a scintillation simulator has been developed. This simulator is divided in three main steps: a scintillation model, a GNSS receiver simulator, and a scintillation estimator.

\section{A. Scintillation model}

The electronic density $\mathrm{Ne}(\vec{r}, t)$, with $\vec{r}$ the vector path and $\mathrm{t}$ the time, is modelled as a stochastic process, where the mean effect $\langle N e(\vec{r}, t)\rangle$ may be given by a traditional ionosphere model, such as NeQuick or Klobuchar, and the dynamic one, reproducing the scintillation due to turbulence in the electronic density, given by a centred random process $\Delta N e(\vec{r}, t)$.

$$
N e(\vec{r}, t)=\langle N e(\vec{r}, t)\rangle+\Delta N e(\vec{r}, t)
$$

The stochastic process $\Delta N e(\vec{r}, t)$ is model thanks to the Schkarofsky spectrum. The link between this spectrum and the amplitude / phase fluctuations of an electromagnetic waves propagating through such turbulent environment is given in [2] [3]. At the end, we define the generic scintillation spectrum of the $\log$ amplitude $W_{\chi}^{3 D}(f)$ and phase $W_{\phi}^{3 D}(f)$ as follow:

$$
\begin{aligned}
& W_{\chi}^{3 D}(f)= \begin{cases}A_{\chi} & \text { if } f<f_{\chi}^{c} \\
A_{\chi}\left(\frac{f}{f_{\chi}^{c}}\right)^{1-P} & \text { if } f>f_{\chi}^{c}\end{cases} \\
& W_{\phi}^{3 D}(f)= \begin{cases}A_{\phi} & \text { if } f<f_{\phi}^{c} \\
A_{\phi}\left(\frac{f}{f_{\phi}^{c}}\right)^{1-P} & \text { if } f>f_{\phi}^{c}\end{cases}
\end{aligned}
$$

4 parameters can define these spectrums: $\left(A_{\chi}, p, f_{\chi}^{c}, f_{\phi}^{c}\right)$ which are respectively the amplitude of the log-amplitude spectrum, the slope, the cut off frequency of the logamplitude spectrum and the cut off frequency of the phase spectrum. Please note that amplitude of the phase spectrum is given by [3]:

$$
A_{\phi}=A_{\chi}\left(\frac{f_{\phi}^{c}}{f_{\chi}^{c}}\right)^{1-P}
$$

Then, by filtering a random Gaussian noise by such spectrum, scintillation time series are generated. An example of FFT (Fast Fourier Transform) processed logamplitude time series is presented Figure 1.

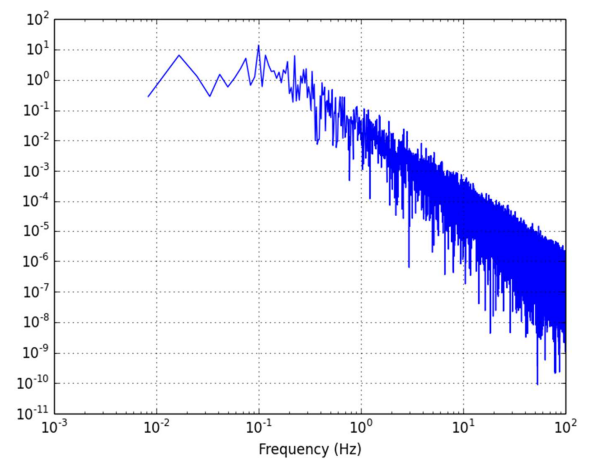

Figure 1: FFT of generated amplitude time series

\section{B. GNSS receiver model}

The GNSS receiver model is based on a post-correlation approach reproducing a GPS C/A signal over $10 \mathrm{MHz}$ bandwidth integrated over 5ms. The implemented DLL / PLL / FLL are described in [6]. The delay and the phase are modeled considering the geometric range between satellite and receiver, and including the phase ionospheric time series generated by the scintillation model. All the other bias (e.g. tropospheric, clock ...) are not modeled as they are out of the scope of the present study. Regarding the amplitude, the log-amplitude time series generated by the scintillation model is used. Finally, the GNSS receiver model is able to provide pseudo range, phase and Doppler estimations during both scintillation and non-scintillation events.

As the signal is integrated other $5 \mathrm{~ms}$, the outputs rate is $200 \mathrm{~Hz}$, which allows a $100 \mathrm{~Hz}$ as maximum span on the spectrum.

\section{Scintillation estimator}

The goal here is to estimate the log-amplitude and the phase scintillation spectrum based on the GNSS observables. To do so, the following procedure has been applied [7].

For the phase processing, the first step is to remove cycle slips by checking the coherency between the phase and the Doppler estimate. If the difference between the estimated Doppler and the phase derivative estimated by the PLL loop is higher than a tolerance of $10 \mathrm{~Hz}$, a cycle slip is assumed, and this portion of the data is removed. The second step is to remove the satellite dynamic from the spectrum. To do so, a Butterworth high pass filter of $6^{\text {th }}$ order is used. The cut off frequencies of the filter are usually between $0.1 \mathrm{~Hz}$ and $0.3 \mathrm{~Hz}$. As a third step, the first second of the post-processed time series of observables is removed in order to avoid filter transition effects. Finally, the spectrum is estimated by the mean of a FFT.

For the amplitude processing, the first step is to transform the post correlated I/Q samples into log-amplitude time series. Then, a "detrending" method is applied which consist in filtering the log-amplitude time series with a Butterworth low pass filter of $6^{\text {th }}$ order with, typical cut off frequencies between $0.1 \mathrm{~Hz}$ and $0.3 \mathrm{~Hz}$, in order to 
extract the signal "template". This template is subtracted to the original log-amplitude time series removing its slow variations. Finally, as for the phase processing, the first second of the post-processed time series of observables is removed. The spectrum is finally estimated by FFT.

The reader is reminded that the scintillation indices $\sigma_{\phi}$ and S4 are the results of the integration of the previously presented spectrums. Thus, spectrum estimation is fully equivalent to scintillation indices estimation.

$$
\begin{aligned}
S 4 & =4 \int W_{\chi}^{3 D}(f) d f \\
\sigma_{\phi} & =\sqrt{\int W_{\sigma}^{3 D}(f) d f}
\end{aligned}
$$

\section{Simulation results}

For each simulation, 300 seconds of GNSS signal have been simulated. For the first 180 s, no scintillation occurs. During this time, we are able to estimate the frequency response of the receiver loops. Then, scintillation occurs between 180 and 300s. The estimation of the scintillation spectrums is done during this second time interval. Figure 2 illustrates this scintillation timing through the observation of the PLL error. The acquisition and phase recovery steps of the PLL can be observed during the first second. Then the PLL reaches it steady state before being perturbed by a phase scintillation event after $\mathrm{t}=180 \mathrm{~s}$.

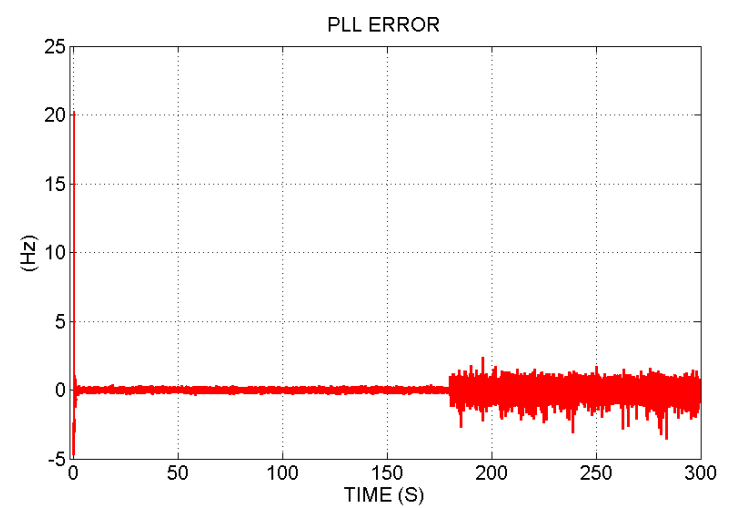

Figure 2: PLL error without $(\mathrm{t}=0: 180 \mathrm{~s})$ and with scintillation $(t=180: 300)$

Table 1 gives typical scintillation parameters extracted from [3] which were used for the next presented simulations.

Table 1: Ionospheric scintillation parameters

\begin{tabular}{|c|c|c|c|}
\hline$A_{\chi}$ & $p$ & $f_{\chi}^{c}(\mathrm{~Hz})$ & $f_{\phi}^{c}(\mathrm{~Hz})$ \\
\hline 20 & 3.5 & 0.1 & 0.01 \\
\hline
\end{tabular}

\section{A. Phase spectrum estimation}

In Figure 3, an example of spectrum estimation for an unrealistic case of $\mathrm{C} / \mathrm{NO}=200 \mathrm{dBHz}$ is presented. The idea is to observe the influence of the loops only on the scintillation spectrum estimation. Figure 3, spectrum named input is estimated directly from the output of the scintillation model, without passing through the GNSS receiver nor the scintillation estimator. It is considered as the reference spectrum and therefore the target of the estimation. The input (with filter) spectrum is estimated from the output of the scintillation model, which goes through the scintillation estimator only. This spectrum is therefore independent from the GNSS receiver behavior, and depends only of the scintillation estimator parametrization. The two other spectrums, no iono and iono, are based on the GNSS receiver outputs which go through the scintillation estimator. The no iono spectrum curve can be interpreted as the frequency response of the receiver, estimated when no scintillation occur (from $2 \mathrm{~s}$ to 180s). The iono spectrum curve is estimated during a single scintillation event. Finally, the asymptotic response of the PLL, and the theoretical pattern of the scintillation are added in dashed line.

In Figure 3, the impact of the PLL bandwidth can be highlighted. Here, a PLL bandwidth of $5 \mathrm{~Hz}$ was used, which affects the slope of the phase spectrum scintillation. The slope of the iono spectrum suddenly changes around $5 \mathrm{~Hz}$, and no longer follows the input spectrum for frequencies higher than $5 \mathrm{~Hz}$. Another important observation is the influence of the high pass filter used in the scintillation estimator which cuts the low frequency part of the spectrum. Before the cut off frequency ( $\mathrm{fc}=0.2 \mathrm{~Hz}$ in this case), all the spectrum going through the scintillation estimator, no iono, iono and input (with filter) curves, are cut.

Figure 4 presents spectrum estimations with a $\mathrm{C} / \mathrm{NO}=$ $45 \mathrm{dbHz}$. In order to minimize the loop bandwidth influence, $10 \mathrm{~Hz}$ bandwidth for the PLL was used. With such a $\mathrm{C} / \mathrm{N} 0$, a double slopes effect can be seen, with a floor between these two slopes. The first slope is clearly the scintillation spectrum, as the input and iono spectrums perfectly match between the cut of frequency $(\mathrm{fc}=0.1 \mathrm{~Hz})$ and the end of the first slope $(\mathrm{f}=0.3 \mathrm{~Hz})$. The $10^{-4}$ floor observed on the spectrum is the noise floor. It can be clearly identified thanks to the no iono spectrum, which is flat over the PLL bandwidth as expected. From this frequency, the scintillation spectrum cannot be estimated anymore as it is below the noise. The last slope is the PLL response, which is not linked to the any atmospheric effect. 


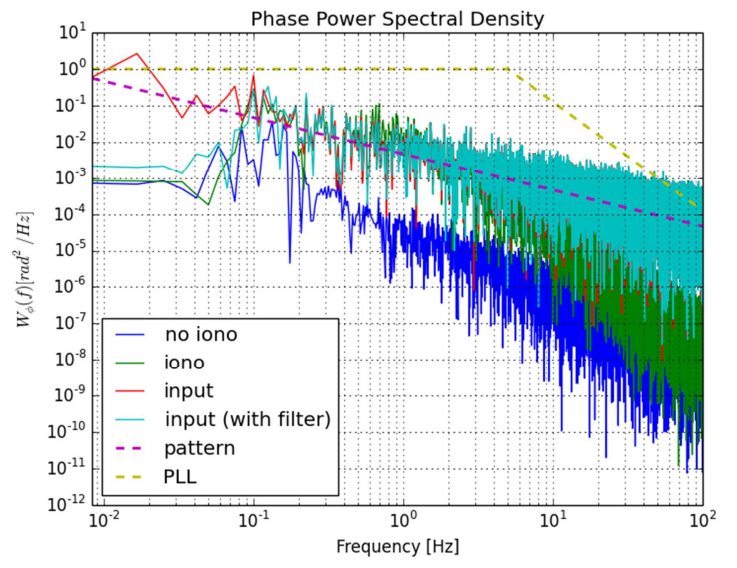

Figure 3: example of phase spectrum estimation, $\mathrm{C} / \mathrm{N} 0=$ $200 \mathrm{dBHz}$

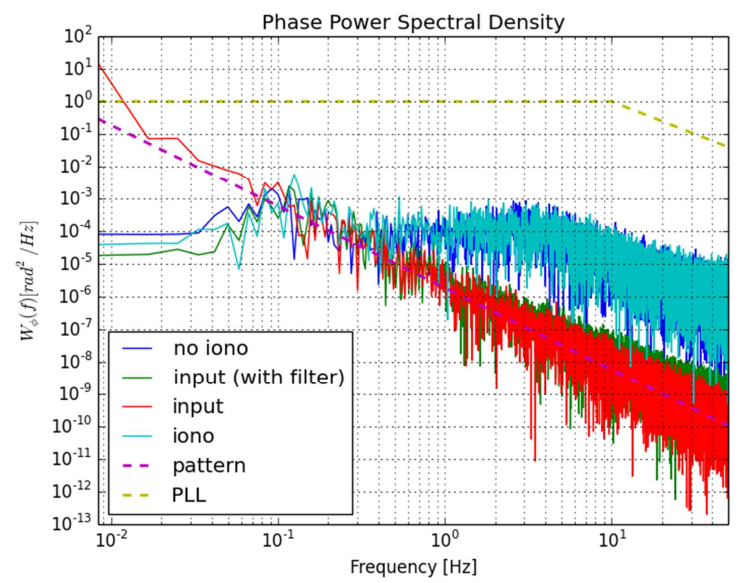

Figure 4: example of phase spectrum estimation, C/N0 = $45 \mathrm{dBHz}$

The consequences on the scintillation indices are the following:

The phase slope should be estimated only in the interval where the effective scintillation can be measured. Thus, the first recommendation is to estimate the slope between the cut off frequency of the high pass filter "fc" and the PLL bandwidth. This recommendation leads to use an fc as small as possible $(0.1 \mathrm{~Hz})$ and a PLL bandwidth as big as possible (BPLL > $15 \mathrm{~Hz}$ ). The reader is reminded that the frequency range is limited by the sampling rate of the output. Consequently, it is not required to use a PLL bandwidth higher than the sampling rate. In the case of low $\mathrm{C} / \mathrm{N} 0$, the frequency of the noise floor (note $\mathrm{F}_{\text {noise }}$ ) should be estimated. Then, the phase slope should be estimated between fc and $F_{\text {noise }}$.

For the $\sigma_{\varphi}$ index, the cut off frequency of the high pass filter "fc" will have a big influence. Indeed, a major part of the energy of the phase spectrum is at low frequency as the cut off frequency of the phase spectrum are usually very small $(\sim 0.01 \mathrm{~Hz})$ [3]. In Figure 5 and Figure 6, simulated and estimated $\sigma_{\varphi}$ are compared considering a cut off frequency for the high pass filter respectively equal to 0.2 and $0.3 \mathrm{~Hz}$. The scintillation parameters are summarizes in table 1 , and a second order PLL with a 10 $\mathrm{Hz}$ bandwidth is used. The $\mathrm{C} / \mathrm{NO}$ is equal to $50 \mathrm{dBHz}$. In these two figures, the following labels are used: the reference $\sigma_{\varphi}$ (labeled ref) is based on the output of the scintillation model, the estimated $\sigma_{\varphi}$ (labeled estimated) is based on the GNSS outputs, the filtered reference $\sigma_{\varphi}$ (labeled ref with filter) is based on the high pass filtered time series from the scintillation model. For the last, note that the GNSS receiver is bypassed, the spectrum estimator influence only is then evaluated. Comparing these two figures, the higher the fc, the larger the estimation error is. We also observe that the estimated and the ref with filter curves are very close, independent of fc. The conclusion is that the observed bias is not coming from the receiver tuning, but from the estimation strategy itself with cuts a major part of the scintillation energy.

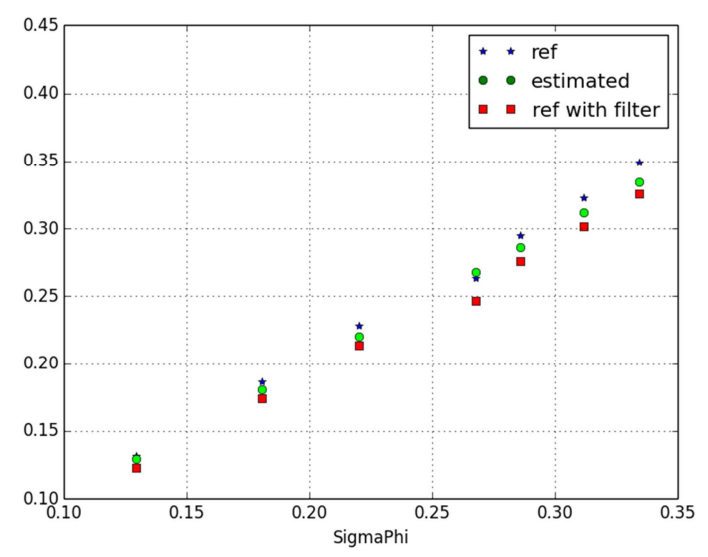

Figure 5: estimated vs simulated $\sigma_{\varphi}$ with $\mathrm{fc}=0.1 \mathrm{~Hz}$

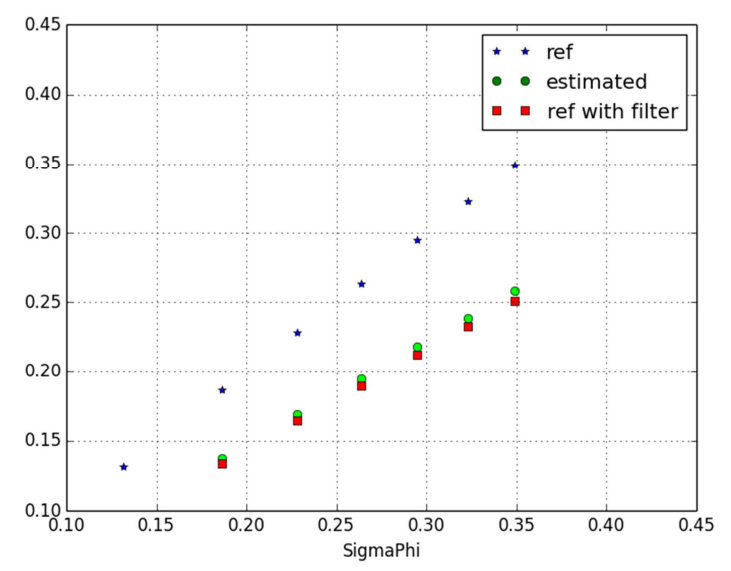

Figure 6: estimated vs simulated $\sigma_{\varphi}$ with $\mathrm{fc}=0.2 \mathrm{~Hz}$

To better highlight the influence of the cut off frequency on the estimated $\sigma_{\varphi}$ index, raw data from a Septentrio GPS receiver placed in polar region (Tromso, Norway) were processed with different fc values. As we can see on Figure $7, \sigma_{\varphi}$ values are strongly affected by the chosen cut off frequency. 


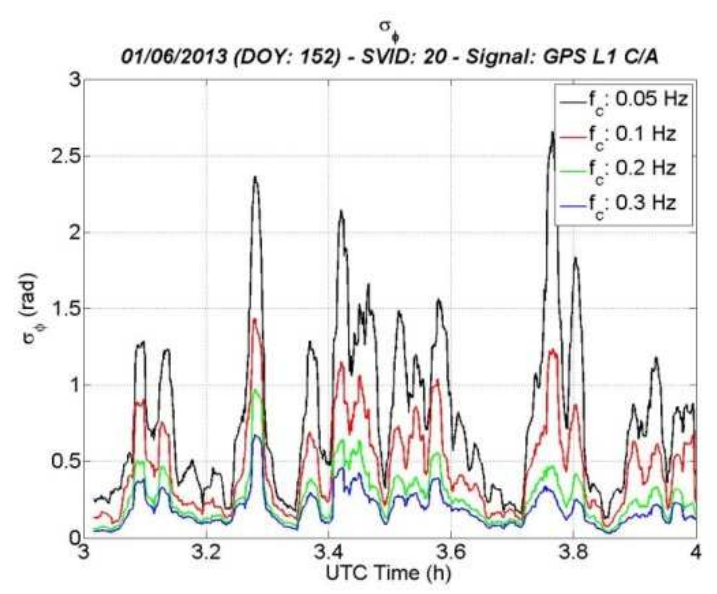

Figure 7: Influence of fc based on raw data from Tromso GNSS receiver, 2013/06/01, PRN 20

The PLL bandwidth influences the high part of the spectrum. However, this part of the spectrum has lower scintillation energy as the phase spectrum is decreasing. Thus, for the $\sigma_{\varphi}$ estimation, which is the integration of the phase spectrum, as stated in Eq.(6), PLL bandwidth has almost no influence. The Figure 8 shows simulations results where different PLL bandwidths were tested. The scintillation parameters are presented in table 1 , the $\mathrm{C} / \mathrm{N} 0$ is equal to $50 \mathrm{dBHz}$ and $\mathrm{fc}=0.2 \mathrm{~Hz}$. As visible, the $\sigma_{\varphi}$ estimations are close to the reference, with a difference of about $0.05 \mathrm{rad}$, independent of the PLL bandwidth.

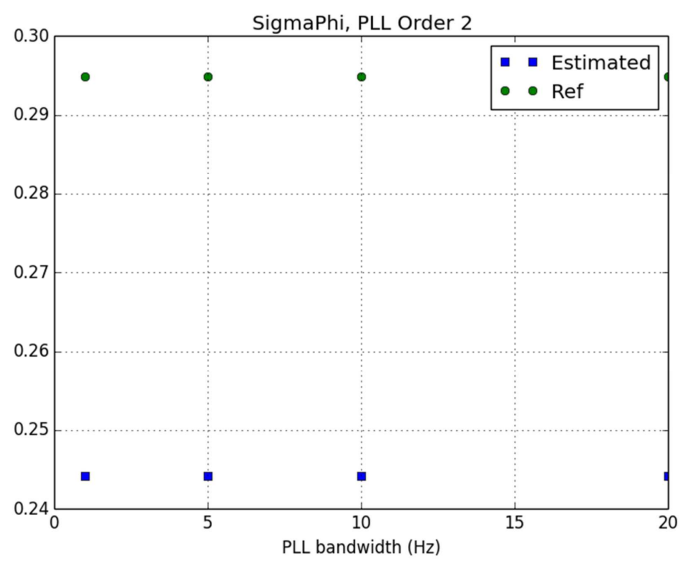

Figure 8: SigmaPhi estimation vs PLL bandwidth

\section{B. Log amplitude spectrum estimation}

The log-amplitude spectrum is based on the I/Q time series, which are almost independent of the DLL and PLL parametrization. As an example, Figure 9 presents reference and estimated spectrums using the same naming convention as in Figure 3. Here, a $1 \mathrm{~Hz}$ bandwidth for both DLL and PLL is used. The filtering effect of both loops is not predominant on the estimated spectrum. As a conclusion, the GNSS receiver tuning seems to have almost no impact on the log-amplitude spectrum estimation. Figure 10 and Figure 11, respectively compare the log-amplitude spectrum slope and the S4 index for both estimated and simulated scenarios with several PLL bandwidths. The ionospheric parameters are the ones in table 1 , and we use $\mathrm{fc}=0.1 \mathrm{~Hz}$ for the "detrending step". The $\mathrm{C} / \mathrm{NO}$ is equal to $50 \mathrm{dBHz}$, and the slopes were estimated from fc to $50 \mathrm{~Hz}$. As visible, the bandwidth does not affect the estimation of the log-amplitude parameters.

The "detrending step" in the log-amplitude spectrum estimation process is similar to a high pass filter as it removes the low frequency part of the spectrum. This similar behavior compared to phase spectrums can be observed Figure 9. However, the cut off frequency of the theoretical log-amplitude spectrum are much higher than the cut off frequency of the theoretical phase spectrum ( $0.1 \mathrm{~Hz}$ ) [3]. Thus, the fc parameters will less affect the $\mathrm{S} 4$ estimation, as less energy is cut. A theoretical comparison, between phase and log-amplitude spectrum, is propose in the annex in order to evaluate the power losses introduced by the high pass filter. It is demonstrated that for $\mathrm{fc}=0.1 \mathrm{~Hz}$, a minimum of $10 \mathrm{~dB}$ on the total phase scintillation power is lost by the high pass filter. For the log-amplitude spectrum, a maximum of $6 \mathrm{~dB}$ is lost.

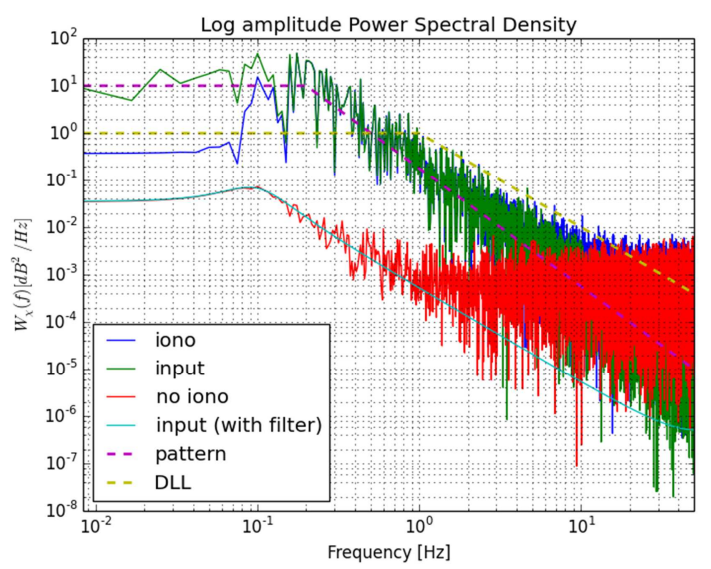

Figure 9: log-amplitude spectrums

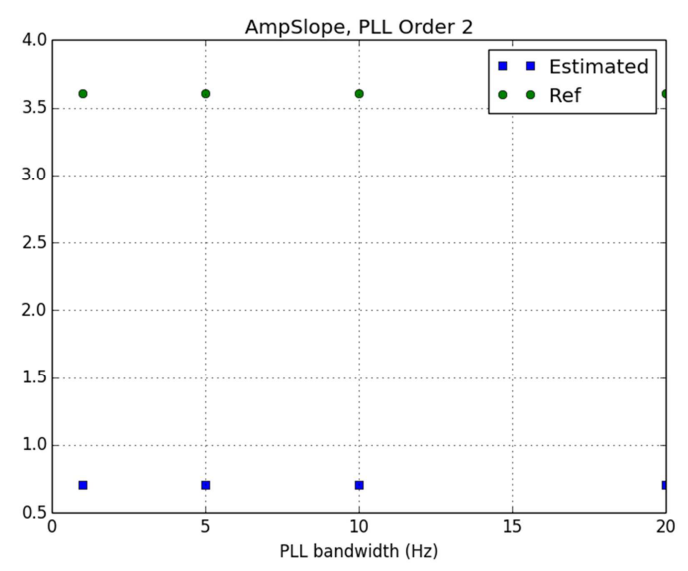

Figure 10: Amplitude slope estimation vs PLL bandwidth 


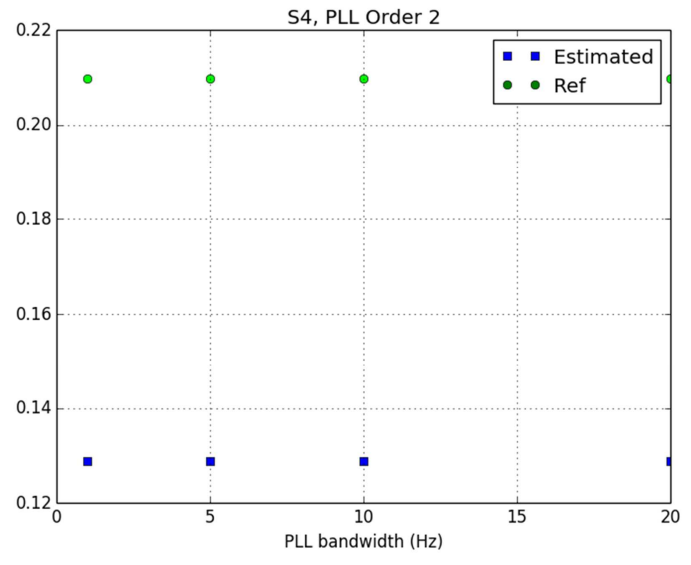

Figure 11: S4 estimation vs PLL bandwidth

To better highlight the influence of the cut off frequency on the estimated S4 index, raw data from a Septentrio GPS receiver placed in polar region (Tromso, Norway) were processed with different fc values. As we can see on Erreur ! Source du renvoi introuvable., S4 values are not affected by the chosen cut off frequency.

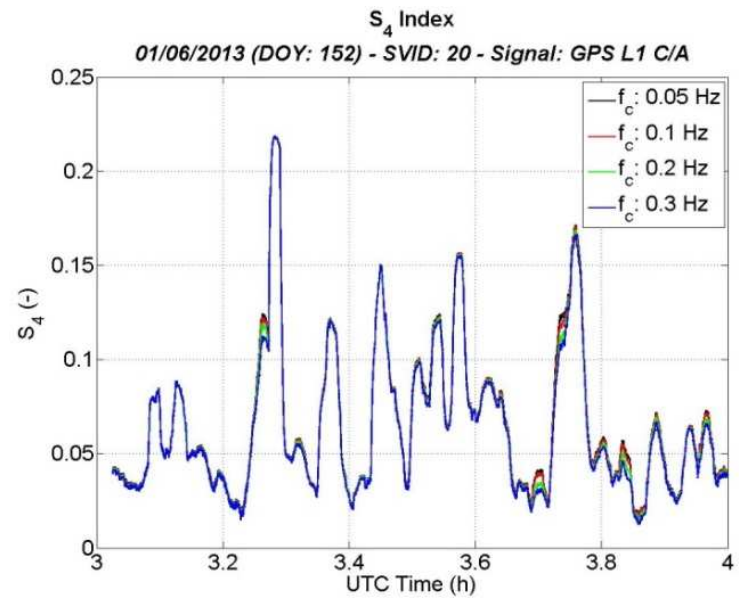

Figure 12: S4 estimation for different fc parameters

Regarding S4index estimation, only noise can affect the log-amplitude spectrum. It will create a floor, which may bias the slope estimation (same as the phase spectrum) and therefore the S4 estimation. Removing the noise influence in S4 estimation is a well-known procedure [7]. However, contrary to the phase spectrum which is affected by loop effects at high frequency, the noise is not cut at high frequency in the log-amplitude spectrum. Thus, the frequency rate, or in other words the frequency range on which to estimate the spectrum, will affect the S4 estimation as the noise contribution will increase with the bandwidth. An example is given in Figure 13, where raw data from a Septentrio GPS receiver placed in polar region (Tromso, Norway) were processed with different sampling rates. The data were collected originally at 100 $\mathrm{Hz}$, and several down sampling were performed to test the $\mathrm{S} 4$ estimator. It can be observed that the S4 index depends on the sampling rate.

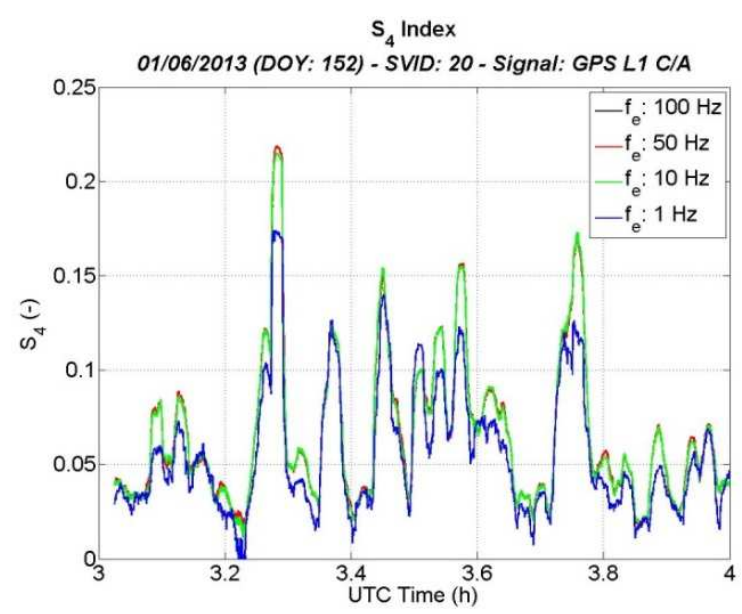

Figure 13: S4 estimation for different sampling rate (denoted fe in the figure)

\section{CONCLUSION}

This paper shows how GNSS receiver tuning and scintillation estimator may bias ionospheric scintillation indices such as $\sigma_{\varphi}, \mathrm{S} 4$ and the log-amplitude and phase slope. This work is based on both simulated and processed data.

As a conclusion on the phase spectrum, its estimation is affected by the GNSS receiver tuning (PLL bandwidth) and by the scintillation estimator (cut off frequency of the high pass filter). The PLL will affect the high frequency component, while the high pass filter will affect the low frequency component.

Regarding the Phase slope estimation, the high frequency component of the spectrum is the most important one. Its estimation may be biased by the PLL bandwidth and the noise threshold. The slope should be estimated on the appropriate portion of the spectrum where the slope remains linear. For high $\mathrm{C} / \mathrm{N} 0$, the slope should be estimated between $\left[\mathrm{fc}, \min \left(\mathrm{B}_{\mathrm{PLL}}, \mathrm{Fe}\right)\right]$, with fc the cut off frequency of the high pass filter, $\mathrm{B}_{\mathrm{PLL}}$ the PLL bandwidth and Fe the sampling rate. For low $\mathrm{C} / \mathrm{N} 0$, the frequency of the noise floor $\left(\mathrm{F}_{\text {noise }}<\min \left(\mathrm{B}_{\mathrm{PLL}}, \mathrm{Fe}\right)\right)$ must be detected, and the slope should be estimate over [fc, $\left.F_{\text {noise }}\right]$.

Regarding the $\sigma_{\varphi}$ index, the low frequency component is important as the theoretical phase spectrum shape is decreasing very fast. Thus, the high pass filter will remove a major part of the scintillation energy from a spectral point of view, which finally affects the $\sigma_{\varphi}$ value. The users are advised that the $\sigma_{\varphi}$ index is strongly linked to the cut off frequency of the high pass filter. On the other hand, the GNSS receiver tuning will almost not affect the $\sigma_{\varphi}$ estimation.

The log-amplitude spectrum is based on the I/Q samples, which are independent from the DLL and PLL parametrization. Finally, the GNSS receiver tuning will have almost no effect on the estimation of S4 and logamplitude slope. The "detreding step" of the scintillation estimator will cut the low frequency component of the log-amplitude spectrum inducing power losses without major consequences. 
In the other hand, as the log-amplitude spectrum is not affected by loops, no filtering effects append at high frequency. Thus, the higher the data rate, the higher the integration frequency range in the spectrum which finally amplifies the noise contribution in the $\mathrm{S} 4$ estimation. As a conclusion, the users should be aware that $\mathrm{S} 4$ values will depend on the sampling rate.

Future work is still to be done in order to provide indices independent from the GNSS receiver tuning and scintillation estimator parametrisation. For example, satellite motion may be suppressed from the phase spectrum with the help of the geometrically computed Doppler. This solution may be substituted to the low pass filter applied during the detrending step, which cuts a large part of the phase spectrum.

\section{ACKNOWLEDGEMENT}

The authors would like to thank NMA and NSC for providing data in polar region.

\section{REFERENCES}

[1] Boscher D, Carvalho F, Fabbro V, Lemorton J, Fleury $\mathrm{R}$ " Modelling ionospheric effects for L band GNSS receivers at high latitudes", Eucap 2014

[2] REPORT ITU-R P.2097, "Transionospheric radio propagation, The Global Ionospheric Scintillation Model (GISM)"

[3] H Galiègue, Ph.D dissertation, «Modélisation des effets des scintillations ionosphériques sur la propagation des ondes éléctromagnétiques en bande L aux latitudes polaires », 2015, Toulouse

[4] Hugues Secretan, Sebastien Rougerie, Lionel Ries, Michel Monnerat, Jérémie Giraud, Roland Kameni, "SAGAIE A GNSS Network for Investigating Ionospheric Behavior in Sub-Saharan Region », Inside GNSS Septembre / October 2014

[5] Y. Béniguel, M. Hernandez-Pajares, A. Garcia-Rigo, R Orus-Perez, R. Prieto-Cerdeira, S. Schlueter, H. Secretan, M. Monnerat, D. Serant «MONITOR IONOSPHERIC MONITORING SYSTEM: GNSS PERFORMANCE ESTIMATION », ENC 2015

[6] D.Kaplan: "Understanding GPS, Principles and Applications", Second Edition

[7] Van Dierendonck A. J., Klobuchar J., And Hua Q. Ionospheric Scintillation Monitoring Using Commercial Single Frequency C/A Code Receivers, in: Proceedings of the $6^{\text {th }}$ Mitigation of Ionospheric Threats to GNSS: an Appraisal of the Scientific and Technological Outputs of the TRANSMIT Project 58

\section{ANNEX}

In this annex, we propose to evaluate to power cut by the high pass filter from a theoretical point of view. To that, we will compute the phase power between 0 and the cut off frequency $f_{c}>f_{\phi}^{c}$. Here, we use the equation (2), (3).

$$
\begin{gathered}
P_{\phi}\left(f_{c}\right)=\int_{0}^{f_{c}} W_{\phi}(f) d f \\
P_{\phi}\left(f_{c}\right)=A_{\phi} f_{\phi}^{c}+\int_{f_{\phi}^{c}}^{f_{c}} A_{\phi}\left(\frac{f}{f_{\phi}^{c}}\right)^{1-P} d f \\
P_{\phi}\left(f_{c}\right)=A_{\phi} f_{\phi}^{c}+\frac{A_{\phi} f_{\phi}^{c}}{2-p}\left[\left(\frac{f_{c}}{f_{\phi}^{c}}\right)^{2-P}-1\right]
\end{gathered}
$$

Please note $\mathrm{p}>3$ [3]. Then let's note the equation as:

$$
P_{\phi}\left(f_{c}\right)=A_{\phi} f_{\phi}^{c}+\frac{A_{\phi} f_{\phi}^{c}}{p-2}\left[1-\left(\frac{f_{\phi}^{c}}{f_{c}}\right)^{p-2}\right]
$$

The total power is given by:

$$
P_{\text {tot }}=\lim _{f_{c} \rightarrow \infty} P\left(f_{c}\right)=A_{\phi} f_{\phi}^{c}+\frac{A_{\phi} f_{\phi}^{c}}{p-2}
$$

In order to evaluate the power cut by the high pass filter, we plot $10 * \log _{10}\left(\frac{P_{t o t}-P_{\phi}\left(f_{c}\right)}{P_{t o t}}\right)$ as a function of fc and for different slop value. As we can see, a minimum of $10 \mathrm{~dB}$ is cut due to the high pass filter (Figure 14) for fc = $0.1 \mathrm{~Hz}$. The same exercise has been done for the logamplitude spectrum. Here, a maximum of $6 \mathrm{~dB}$ may be lost if we use $\mathrm{fc}=0.1 \mathrm{~Hz}$.

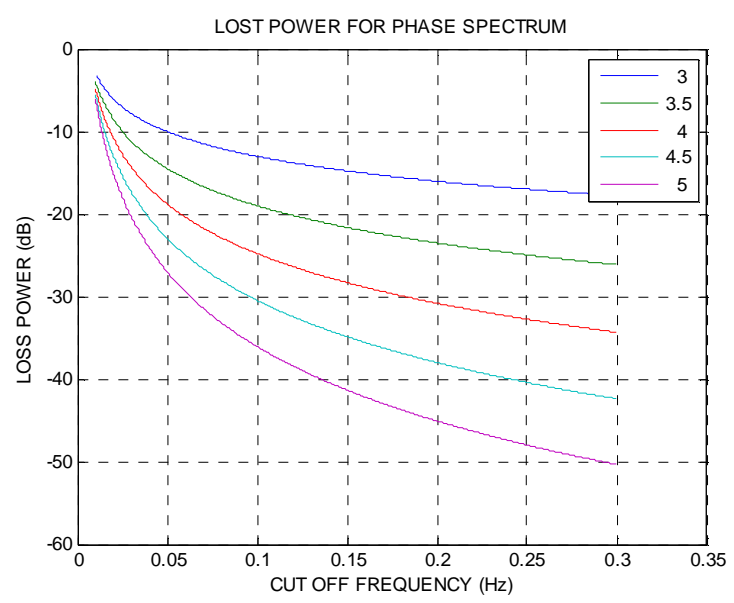

Figure 14: Power lost due to high pass filter for phase spectrum 


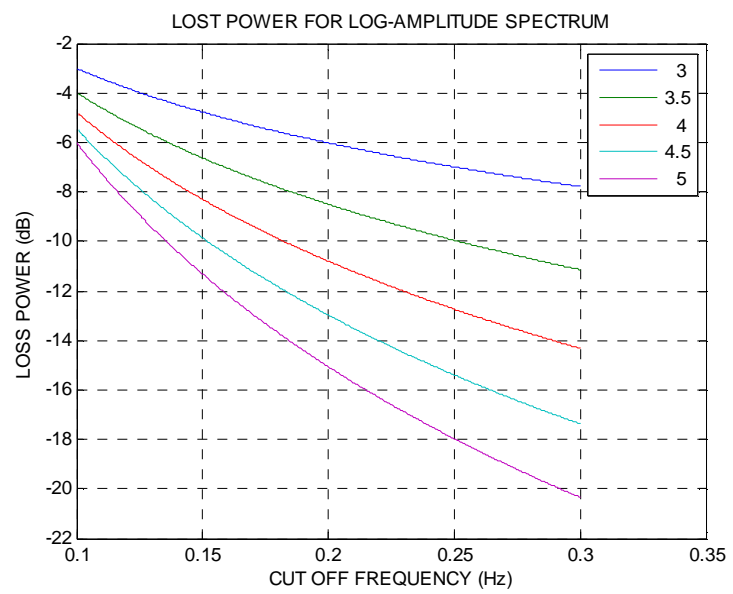

Figure 15: Power lost due to high pass filter for logamplitude spectrum 